Polymer Journal, Vol. 39, No. 10, pp. 1001-1010 (2007)

(C) 2007 The Society of Polymer Science, Japan

\title{
A Numerical Modeling of Dynamic Curing Process of Tire by Finite Element
}

\author{
Xiangqiao $\mathrm{YAN}^{\dagger}$ \\ Research Laboratory on Composite Materials Harbin Institute of Technology, Harbin, 150001, P. R. China
}

(Received December 4, 2006; Accepted March 22, 2007; Published August 7, 2007)

\begin{abstract}
In this paper, a simulator for the tire curing processes is developed based on the finite element method of an axisymmetric heat transfer problem for composite materials. The anisotropy of heat transfer properties of composite materials, the dependence of properties of rubber compounds on the temperature and/or the extent of cure, the time-varying boundary conditions which include the cooldown of the tire out of the press and the rigorous cure kinetic models are taken into account. The numerical simulation results of a truck tire curing process show that the simulator successfully describes the variation trends in temperature and in state of cure with the tire curing process. It also serves as the core module of an optimization algorithm, which is being developed for the tire curing and rubber formulation designs. [doi:10.1295/polymj.PJ2006182]

KEY WORDS Tire / Cure / Finite Element /
\end{abstract}

The curing process is the final step in tire manufacturing whereby a green tire built from layers of rubber compounds and fiber/rubber composites is formed to the desired shape in a press. A schematic cross section of a dome-type cure press is shown in Figure 1. In the press, heat is transferred to the green tire from the mold and the bladder, which are kept at higher temperatures by circulated cure media like steam, hot water, etc. The transferred heat provokes the curing reaction of the rubber compounds, thus converting the compounds to a strong, elastic material to meet tire performance needs.

The curing process is energy consuming and has a strong effect on the product quality. Major operating variables are the conditions of the supplied media, which are to be varied according to prescribed cure steps. To optimize the cure steps for different compounds of various dimensions requires proper evaluation of the time-dependent temperature distribution of various compounds in the tires. The conventional method is to directly measure the temperature-time profiles using thermocouples inserted into various parts of a green tire and then to convert the measured profiles to the state of cure (SOC). But this method is costly and very time-consuming, hence as an alternative, an effective computer simulator of the process has been sought by this industry. ${ }^{8}$

Several studies have been reported on the numerical simulation of tire curing processes. Ambelang and Prentice $^{3}$ outlined a finite difference technique for calculating the state of cure in a vulcanizing tire, but they assumed constant thermal diffusivity, constant rate of heat generation due to vulcanization, and constant boundary conditions. The capability of their model was somewhat extended by Prentice and Williams ${ }^{4}$ by considering temperature-dependent thermal conductivity, chemical generated heat production as a function of temperature and the SOC, and time varying boundary conditions. But it was still assumed that the tire consists of uniform material. Another approach based on the finite difference method was reported by Schlanger ${ }^{5}$ who developed a one-dimensional model, which reduced a complex tire section to a quasi-equivalent slab.

Taking into account of the complex shape and multilayered structure of a tire, a finite element approach is believed to provide better accuracy and versatility than a finite difference method. Hubbard and Simpson ${ }^{6}$ used the finite element method, but they did not consider the cure during the cooldown in the air after the tire is taken out of the press. In 1991, Toth ${ }^{7}$ used a commercial finite element code, ABAQUS, in conjunction with a user subroutine, to solve the coupled heat transfer and cure problem. A fairly general model was used to describe the dependence of rubber compound properties on temperature and the SOC. Rigorous cure kinetic models developed in the literature were also used in the simulation. In a recent paper, Han and his co-authors ${ }^{8}$ developed an in-house simulator for the tire curing process. They considered the cooldown of the tire out of the press and the whole press assembly, including the bladder and the mold was taken as the domain of analysis. In summary, these previous studies seem to be lacking in considering the anisotropy of heat transfer properties of fiber/rubber composite materials. Generally speaking, the transfer heat properties of composite materials are anisotropic. ${ }^{1}$

${ }^{\dagger}$ To whom correspondence should be addressed (E-mail: yanxiangqiao@hotmail.com). 


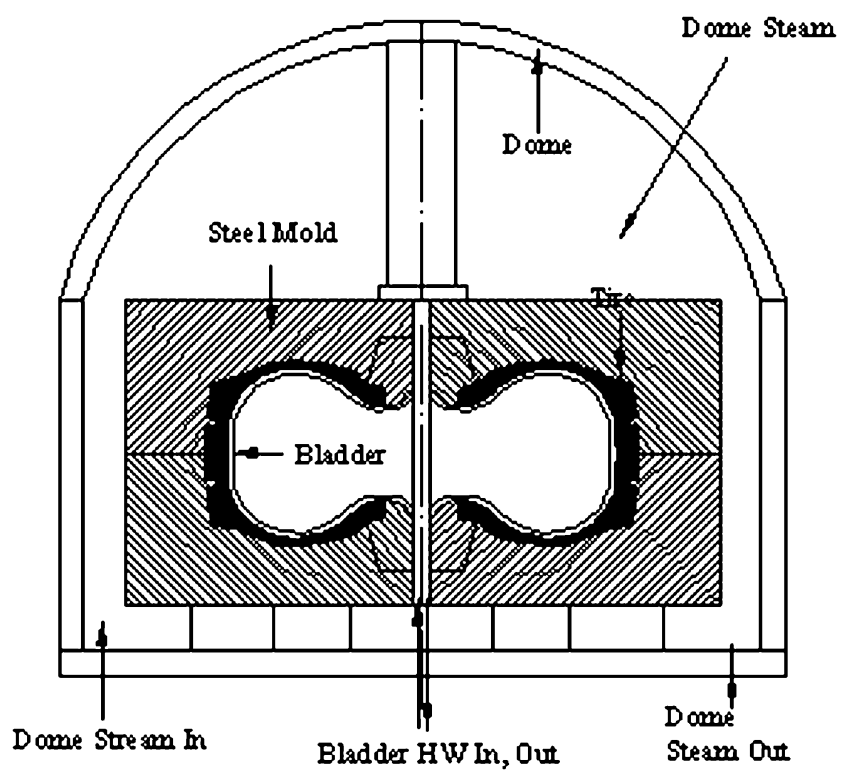

Figure 1. Schematic of tire curing equipment.

The purpose of this study is to present our effort to develop an-house simulator for the tire curing process. The simulator employs the finite element method as the backbone algorithm. It addresses most of the modeling aspects of tire curing process as follows:

1. Anisotropy of heat transfer properties of composite materials.

2. Tires exhibit complicated layouts of different rubber compound layers and rubber/fiber layers.

3. The actual cure steps are input as time-varying boundary condition.

4. The entire cure cycle covers the cooldown of the tire out of the press.

5. The properties of rubber compounds are dependent on the temperature and the SOC.

6. Rigorous cure kinetic models are employed for proper evaluation of the heat generation as well as the SOC.

\section{THEORETICAL FOUNDATION OF TIRE CURING MODELING}

\section{Heat Transfer Equation}

Heat transfer of tire cure can be seen as an axisymmetric problem which can be described in the following equation ${ }^{1}$ expressed in terms of heat current densities, $\mathrm{q}_{\mathrm{r}}, \mathrm{q}_{\mathrm{z}}$ :

$$
\rho c \frac{\partial T}{\partial t}+\frac{\partial}{\partial z}\left(q_{z}\right)+\frac{1}{r} \frac{\partial}{\partial r}\left(r q_{r}\right)-Q=0
$$

in which $Q$ is an interior heat source density, $\rho$, a material density, $c$, a material heat ratio, $T$ a temperature, and $t$, a time.

Boundary conditions of Eq (1) are

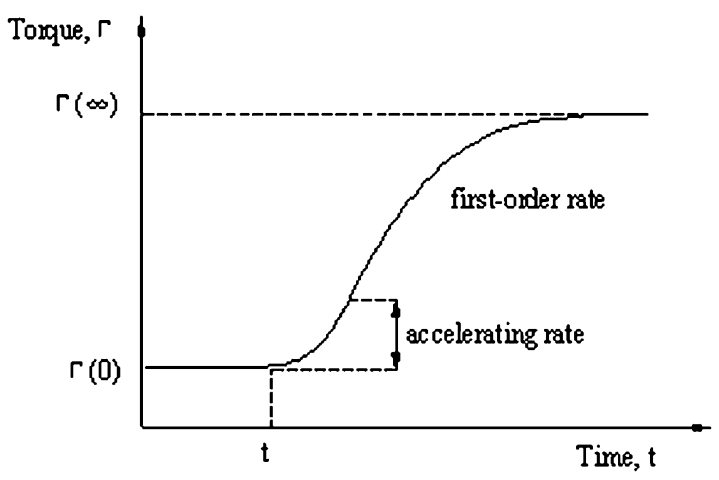

Figure 2. A typical rheometer curve.

$$
\begin{array}{ll}
T(r, z)=f_{a}(r, z, t) & r, z \subset \Gamma_{1} \\
q_{r} n_{r}+q_{z} n_{z}=-q_{a}(r, z, t) & r, z \subset \Gamma_{2} \\
q_{r} n_{r}+q_{z} n_{z}=h\left(T-T_{a}\right) & r, z \subset \Gamma_{3}
\end{array}
$$

where $\Gamma_{1}, \Gamma_{2}$ and $\Gamma_{3}$ are, respectively, the first, second and third boundary conditions; $f_{a}(r, z, t)$ is a known boundary temperature function; $q_{a}(r, z, t)$ is a known boundary heat current density function; $n_{z}, n_{r}$ are normal directional cosines on boundaries; $h$ is a boundary transfer coefficient; and $T_{a}$ is an environment temperature.

It is assumed generally that the thermal conductive properties of composite materials are uniform and transversely isotropic, and are characterized by means of the longitudinal ( $z$-axis direction) and transverse ( $r$ axis direction) thermal conductive coefficient $\lambda_{\mathrm{z}}$ and $\lambda_{\mathrm{r}}$. The Fourier thermal transfer law can then be written as [1]

$$
\left\{\begin{array}{l}
q_{r} \\
q_{z}
\end{array}\right\}=-\left[\begin{array}{cc}
\lambda_{r} & 0 \\
0 & \lambda_{z}
\end{array}\right] \cdot\left\{\begin{array}{c}
\frac{\partial T}{\partial r} \\
\frac{\partial T}{\partial z}
\end{array}\right\}
$$

\section{Cure Reaction Kinetics}

Cure reaction is a process of chemically producing network junctures by the insertion of cross-links between polymer chains. The SOC denotes the extent of the reaction and is determined by measuring the rubber properties dependent on the cross-link density. The most popular and practical method is based on the rheometry whereby time-varying torque required to maintain a given dynamic strain on a vulcanizing rubber specimen is recorded.

Figure 2 illustrate a typical torque $(\Gamma)$ versus time $(t)$ curve of a rubber compound obtained in the rheometry method. There is usually an induction period $\left(t_{i}\right)$ during which little change in torque is observed. A relatively abrupt rise in torque occurs next, characterized by an accelerating slope. The cure reaction kinetics model used in the present paper is ${ }^{7}$ 


$$
\frac{d X}{d t}=K X^{\frac{1}{2}}(1-X) \quad\left(t \geq t_{i}\right)
$$

where

$$
\begin{gathered}
K=K_{0} \operatorname{Exp}\left(-E_{a} / R T\right) \\
t_{i}=t_{i 0} \exp \left(E_{i} / R T\right)
\end{gathered}
$$

where $K$ denotes the rate constant, $t_{i}$ the induction period, $K_{0}$ frequency factor for rate constant of cure reaction, $E_{a}$ activation energy for rate constant of cure reaction, $E_{i}$ activation energy for induction period of cure reaction and $R$ ideal gas constant, $8.314(\mathrm{~J} /$ mol K).

The solution of Eq (6) with $K$ and $t_{i}$ given in Eqs (7) and (8) is obtained as

$$
X(t)=\left\{\left\{\frac{1-\operatorname{Exp}\left[-\int_{t_{i}}^{t} K_{0} \operatorname{Exp}\left[-\frac{E_{a}}{R T(\tau)}\right] d \tau\right]}{1+\operatorname{Exp}\left[-\int_{t_{i}}^{t} K_{0} \operatorname{Exp}\left[-\frac{E_{a}}{R T(\tau)}\right] d \tau\right]}\right\}^{2} \quad t \geq t_{i}\right.
$$

where $t_{i}$ is such that

$$
t_{i 0}=\int_{0}^{t_{i}} \operatorname{Exp}\left[-\frac{E_{a}}{R T(\tau)}\right] d t
$$

For the tire cure considered in this paper, the interior heat source density in Eq (1) is given by

$$
Q=\rho(-\Delta H) \frac{d X}{d t}
$$

which is in fact the rate of heat generation accompanying the vulcanization of rubber compounds. It depends on the heat of vulcanization $\Delta H$ and the rate of reaction, which is measured in terms of the SOC $X$.

\section{Rubber Compound Properties}

The three thermal properties, the thermal conductivity $\lambda$, the material density $\rho$ and the specific heat $c_{\mathrm{p}}$, of rubber compounds involved in the tire curing modeling are dependent on temperature and/or the SOC. In this paper, the dependency of these properties are modeled as follows: ${ }^{7,8}$

$$
\begin{aligned}
& \lambda(T)=a+b T \\
& \rho(X)=(1-X) \rho_{u}+X \rho_{c} \\
& c_{p}(T, X)=(1-X) c_{p u}(T)+X c_{p c}(T)
\end{aligned}
$$

where

$$
\begin{aligned}
& c_{p u}(T)=a_{u}+b_{u} T \\
& c_{p c}(T)=a_{c}+b_{c} T
\end{aligned}
$$

where the subscript $u$ indicates an "uncured state" $(X=0)$ and $c$ a "fully cured" state $(X=1)$. The coefficients in Eqs (12), (15) and (16) are determined from experiments. ${ }^{8}$

\section{FINITE ELEMENT FORMULATION OF A HEAT TRANSFER PROBLEM FOR AXISYMMETRIC COMPOSITE MATERIALS STRUCTURES}

By using a weighted residual method [2], in this section, the finite element formulation for the heat transfer problem expressed in terms of Eqs (1)-(4) is carried out.

Let

$$
T=T\left(r, z, t, T_{1}, T_{2}, \ldots, T_{n}\right)
$$

be a trial temperature field function which satisfies the known temperature boundary condition on $\Gamma_{1}$. $T_{1}, T_{2}, \ldots, T_{n}$ in (17) are unknown constants. The residual functions can be obtained by substituting $T$ in (17) into Eqs (1-4)

$$
\left\{\begin{array}{l}
R_{\Omega}=\rho c r \frac{\partial T}{\partial t}+\frac{\partial}{\partial r}\left(r q_{r}\right)+\frac{\partial}{\partial z}\left(r q_{z}\right)-r Q \\
R_{\Gamma_{1}}=0 \\
R_{\Gamma_{2}}=-\left(q_{r} n_{r}+q_{z} n_{z}\right)-q_{a}(r, z, t) \\
R_{\Gamma_{3}}=h\left(T-T_{a}\right)-\left(q_{r} n_{r}+q_{z} n_{z}\right)
\end{array}\right.
$$

By the weighted residual method, we have

$$
\int_{\Omega} R_{\Omega} u d \Omega+\int_{\Gamma_{2}} R_{\Gamma_{2}} v d \Gamma+\int_{\Gamma_{3}} R_{\Gamma_{3}} w d \Gamma=0
$$

in which $u, v$ and $w$ are, respectively, the weighted functions in the interior $\Omega$ and on boundaries $\Gamma_{2}$ and $\Gamma_{3}$.

The boundary condition on fixed temperature boundary $\Gamma_{1}$ can be treated: (1). As mentioned above, let the trial function satisfy the fixed temperature boundary $\Gamma_{1}$, the remainder being zero; (2). The fixed temperature boundary $\Gamma_{1}$ is dealt with by means of the third boundary condition by letting the boundary heat transfer coefficient $h$ be infinite. Thus the first boundary condition does not occur in the equation (19).

According to finite element method, the regime $\Omega$ is separated into finite elements, and the temperature field function $T$ in each element is interpolated by its nodal temperature $T_{\mathrm{i}}$

$$
T=\sum_{i=1}^{n^{e}} N_{i}(r, z) T_{i}=N \cdot T^{e}
$$


where

$$
\begin{aligned}
N & =\left[N_{1}, N_{2}, \cdots, N_{n^{e}}\right] \\
T^{e} & =\left[T_{1}, T_{2}, \cdots, T_{n^{e}}\right]^{T}
\end{aligned}
$$

where $n^{e}$ is the number of nodes in an element, $N_{i}(r, z)$ is the interpolation function corresponding to the node $i$.

According to the weighted residual method, the weighted function in the regime $\Omega$ is chosen as

$$
\mathrm{u}=\mathrm{N}_{\mathrm{i}}
$$

and the weighted functions $v, w$ on boundaries $\Gamma_{2}$ and $\Gamma_{3}$ are chosen as

$$
w=v=r u=r N_{i}
$$

Substituting (23) and (24 into Eq (19, we can get

$$
\begin{gathered}
\int_{\Omega}\left[\frac{\partial}{\partial r}\left(r q_{r}\right)+\frac{\partial}{\partial z}\left(r q_{z}\right)-r Q+\rho c r \frac{\partial T}{\partial t}\right] N_{i} d \Omega \\
\quad+\int_{\Gamma_{3}}\left[h\left(T-T_{a}\right)-\left(q_{r} n_{r}+q_{z} n_{z}\right)\right]\left(r N_{i}\right) d \Gamma \\
\quad+\int_{\Gamma_{2}}-\left(q_{r} n_{r}+q_{z} n_{z}+q_{a}\right)\left(r N_{i}\right) d \Gamma=0
\end{gathered}
$$

Using the Green's formula, we have

$$
\begin{aligned}
& \int_{\Omega}\left[N_{i} \frac{\partial}{\partial r}\left(r q_{r}\right)+N_{i} \frac{\partial}{\partial z}\left(r q_{z}\right)\right] d \Omega \\
& =\int_{\Omega}\left[\frac{\partial}{\partial r}\left(N_{i} r q_{r}\right)+\frac{\partial}{\partial z}\left(N_{i} r q_{z}\right)\right] d \Omega \\
& -\int_{\Omega}\left[\frac{\partial N_{i}}{\partial r}\left(r q_{r}\right)+\frac{\partial N_{i}}{\partial z}\left(r q_{z}\right)\right] d \Omega \\
& =\oint_{\Gamma}\left[\left(N_{i} r q_{r}\right) n_{r}+\left(N_{i} r q_{z}\right) n_{z}\right] d \Gamma \\
& -\int_{\Omega}\left[\frac{\partial N_{i}}{\partial r}\left(r q_{r}\right)+\frac{\partial N_{i}}{\partial z}\left(r q_{z}\right)\right] d \Omega
\end{aligned}
$$

By substituting (26) into Eq (25), Eq (25) can be simplified as

$$
\begin{aligned}
- & \int_{\Omega}\left[\frac{\partial N_{i}}{\partial r}\left(r q_{r}\right)+\frac{\partial N_{i}}{\partial z}\left(r q_{z}\right)\right] d \Omega \\
& +\int_{\Omega}\left(\rho c r \frac{\partial T}{\partial t}-r Q\right) N_{i} d \Omega+ \\
& \quad \int_{\Gamma_{3}} h\left(T-T_{a}\right) r N_{i} d \Gamma-\int_{\Gamma_{2}} q_{a} r N_{i} d \Gamma=0
\end{aligned}
$$

Using the formula (5) and introducing the following symbols

$$
K_{i j}^{e}=\int_{\Omega^{e}}\left[r \frac{\partial N_{i}}{\partial r}\left(\lambda_{r} \frac{\partial N_{j}}{\partial r}\right)+r \frac{\partial N_{i}}{\partial z}\left(\lambda_{z} \frac{\partial N_{j}}{\partial z}\right)\right] d \Omega
$$

$$
\begin{aligned}
H_{i j}^{e} & =\int_{\Gamma_{3}^{e}} h r N_{i} N_{j} d \Gamma \\
P_{Q_{i}}^{e} & =\int_{\Omega^{e}} Q r N_{i} d \Omega \\
P_{H_{i}}^{e} & =\int_{\Gamma_{3}^{e}} h T_{a} r N_{i} d \Gamma \\
P_{q_{i}}^{e} & =\int_{\Gamma_{2}^{e}} q_{a} r N_{i} d \Gamma \\
C_{i j}^{e} & =\int_{\Omega^{e}} \rho c r N_{i} N_{j} d \Omega
\end{aligned}
$$

then Eq (27) can be represented as

$$
C \dot{T}+K T=P
$$

where $K, P$ and $C$ are, respectively, the heat transfer stiffness matrix, the temperature load vector and the heat capacity matrix:

$$
\begin{aligned}
K_{i j} & =\sum_{e} K_{i j}^{e}+\sum_{e} H_{i j}^{e} \\
P_{i} & =\sum_{e} P_{Q i}^{e}+\sum_{e} P_{H i}^{e}+\sum_{e} P_{q i}^{e} \\
C_{i j} & =\sum_{e} C^{e}{ }_{i j}
\end{aligned}
$$

Here $\sum_{e}$ indicates to sum for all elements.

\section{NUMERICAL VALIDATION}

Using the finite element formulation of a heat transfer problem for asymmetric composite structures described above, a finite element code is developed in this investigation. In this code, a quadrilateral element with four nodes and a triangle element with three nodes and a backward difference formula for a time $t$ are used.

In order to illustrate the accuracy of the finite element formulation presented in this paper and the effect of material thermal conductive anisotropy on temperature field, some examples are given in this section. Heat generation is not considered here.

\section{Example 1}

In this subsection, we consider a cylinder with a circular section whose inner radius $\left(r_{\mathrm{i}}\right)$ and outer radius $\left(r_{\mathrm{o}}\right)$ are $0.30 \mathrm{~m}$ and $0.45 \mathrm{~m}$, respectively. The cylinder height $H$ is $0.30 \mathrm{~m}$. The inner surface of the cylinder is a constant temperature boundary whose temperature is $800^{\circ} \mathrm{C}$. The other boundary surfaces are convective boundaries, with environment temperature $T_{\mathrm{a}}=20^{\circ} \mathrm{C}$ and convection heat transfer coefficient $h=150\left[\mathrm{~W} / \mathrm{m}^{2} \cdot{ }^{\circ} \mathrm{C}\right]$. Let the transverse $(r-$ axis direction) thermal conductive coefficient $\lambda_{r}=$ 
$10\left[\mathrm{~W} / \mathrm{m} \cdot{ }^{\circ} \mathrm{C}\right]$. Also, let the ratio of the transverse thermal conductive coefficient $\lambda_{r}$ to the longitudinal $(z-$ axis direction) $\lambda_{\mathrm{z}}$ be ratio $=\lambda_{r} / \lambda_{\mathrm{z}}$. Here, the ratio $=$ $\lambda_{r} / \lambda_{\mathrm{z}}$ is taken different values to reveal the effect of the thermal conductive anisotropy on steady temperature field.

Obviously, symmetric condition can be used. Figure 3 shows a finite element mesh with 121 nodes and 100 elements. The boundaries $\mathrm{AD}, \mathrm{AB}$ in Figure 3 are taken respectively constant temperature and insulating temperature and boundaries BC and $\mathrm{CD}$ are taken a convection heat transfer boundary. Figure $4(\mathrm{a}-\mathrm{f})$ shows the present numerical results of steady temperature fields as the thermal conductive coefficient ratio ratio $=\lambda_{r} / \lambda_{\mathrm{z}}$ varies. It can be seen from Figure 4 that the thermal conductive anisotropy has an important effect on temperature fields.

\section{Example 2}

Here, we consider the cylinder mentioned in example 1 but the cylinder height is assumed to be infinite. Obviously, temperature fields on various sections in the region far away from the two ends of the cylinder are same. A segment of height $=0.15 \mathrm{~m}$ in the region far away from the two ends of the cylinder is taken consideration here. The boundaries $\mathrm{AB}$ and $\mathrm{CD}$ in

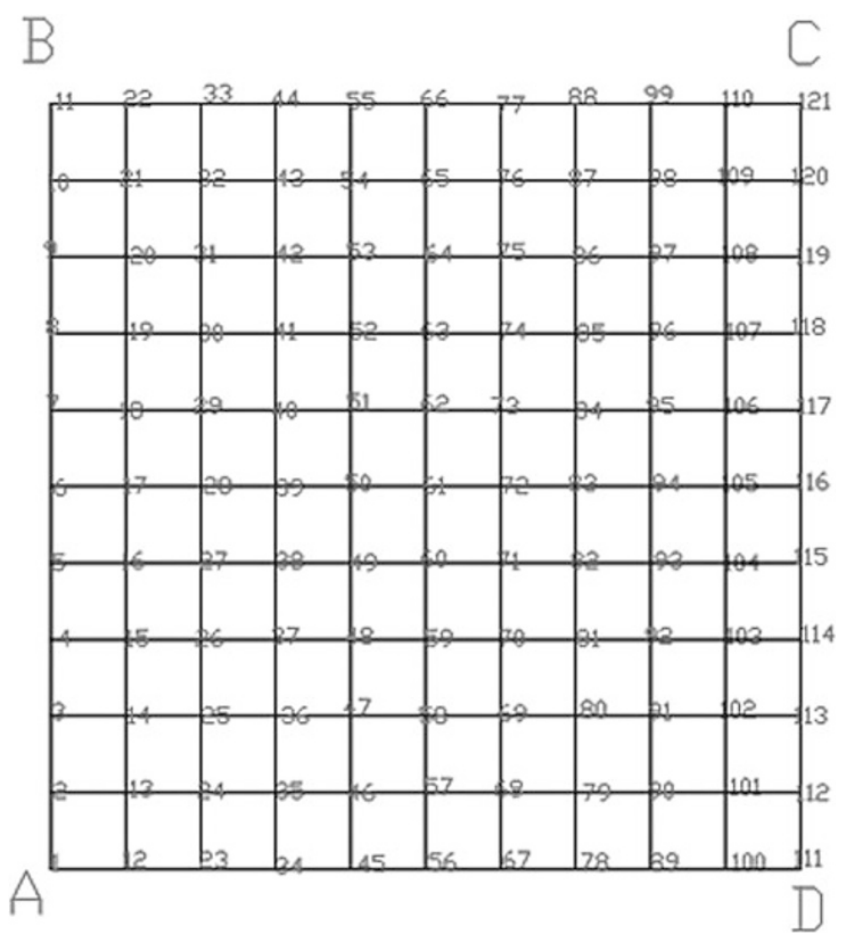

Figure 3. Finite element mesh (including node numbers).

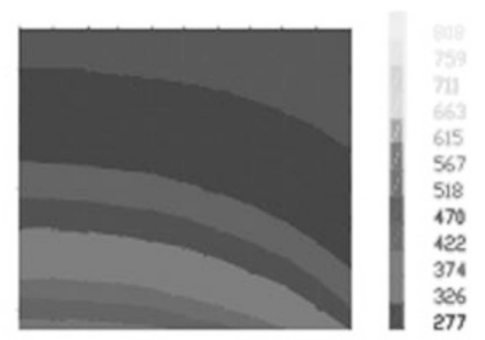

(b) $\left(\lambda\left(d_{2}=0.25\right)\right.$

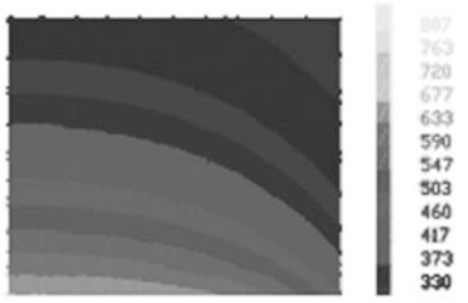

(d) $(d, d,=1)$

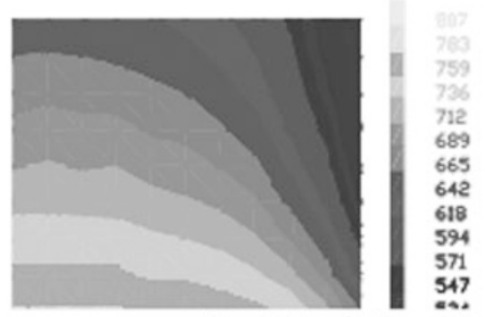

(f) $\left(d, d_{2}=10\right)$

Figure 4. Variation of steady temperature fields with the thermal conductive coefficient ratio $k_{r} / k_{\mathrm{z}}$. 


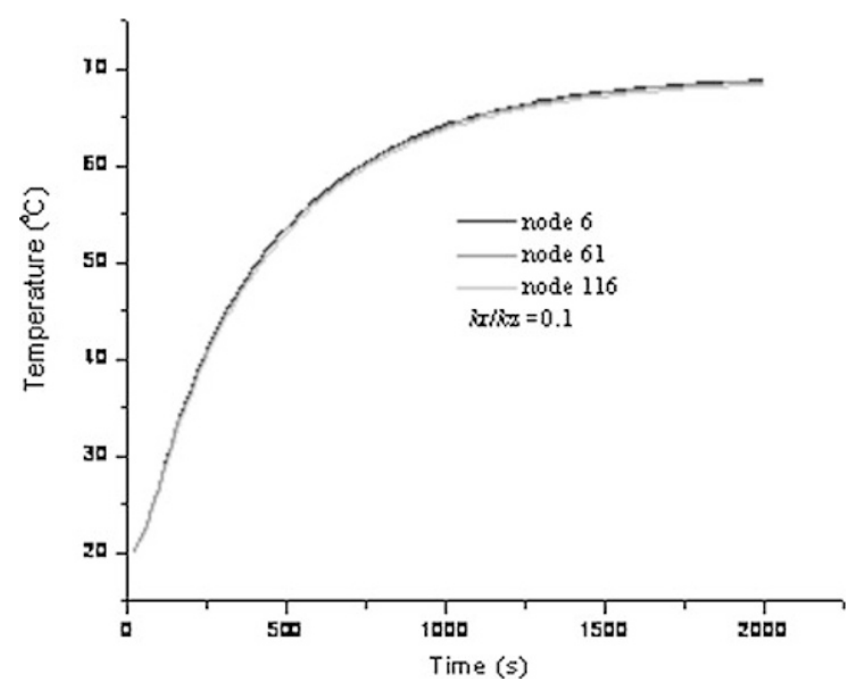

(a)

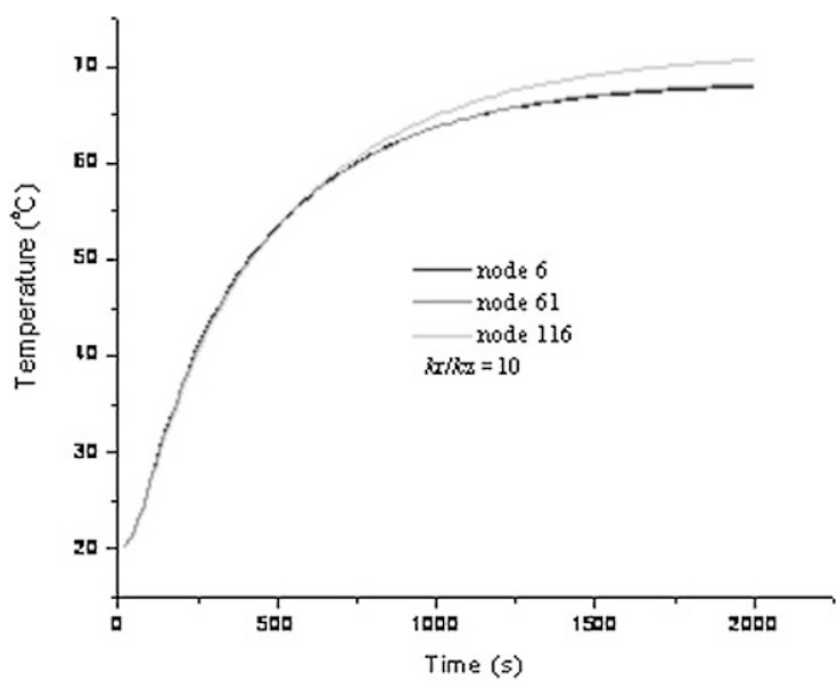

(c)

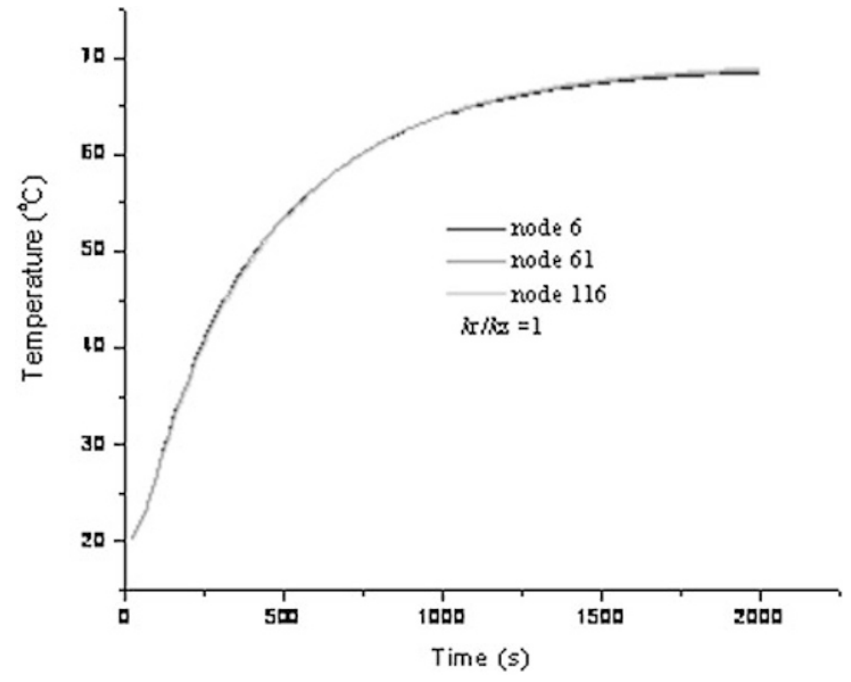

(b)

Figure 5. Variation of transient temperature of nodes 6,61 and 116 with ratio $\lambda_{r} / \lambda_{\mathrm{z}}$.

Figure 3 are taken insulating temperature and the boundaries $\mathrm{AD}$ and $\mathrm{BC}$ are taken respectively constant temperature $\left(100^{\circ} \mathrm{C}\right)$ and convection heat transfer boundaries (convection heat transfer coefficient $h=150\left[\mathrm{~W} / \mathrm{m}^{2} \cdot{ }^{\circ} \mathrm{C}\right]$, surrounding temperature $T_{\mathrm{a}}=$ $20^{\circ} \mathrm{C}$ ). The initial temperature in the cylinder is taken $20^{\circ} \mathrm{C}$. Let the transverse thermal conductive coefficient $\lambda_{r}=10\left[\mathrm{~W} / \mathrm{m} \cdot{ }^{\circ} \mathrm{C}\right]$. Also, let the longitudinal thermal conductive coefficient $\lambda_{\mathrm{z}}=1,10,100[\mathrm{~W} /$ $\left.\mathrm{m} \cdot{ }^{\circ} \mathrm{C}\right]$ and the product of material density $(\rho)$ and material specific heat $(c)$ is taken $10^{6}\left[\mathrm{~W} \cdot \mathrm{s} / \mathrm{m}^{3} \cdot{ }^{\circ} \mathrm{C}\right]$. Obviously, transient temperature fields in the cylinder are one-dimensional, i.e., $T=T(r, t)$, and their results are not dependent on ratio $\lambda_{r} / \lambda_{\mathrm{z}}$.

The finite element mesh used is shown in Figure 3. Figure $5(\mathrm{a}-\mathrm{c})$ show a transient temperature comparison among nodes 6,61 and 116 as ratio $\lambda_{r} / \lambda_{z}$ varies. Figure 6 gives a transient temperature comparison of

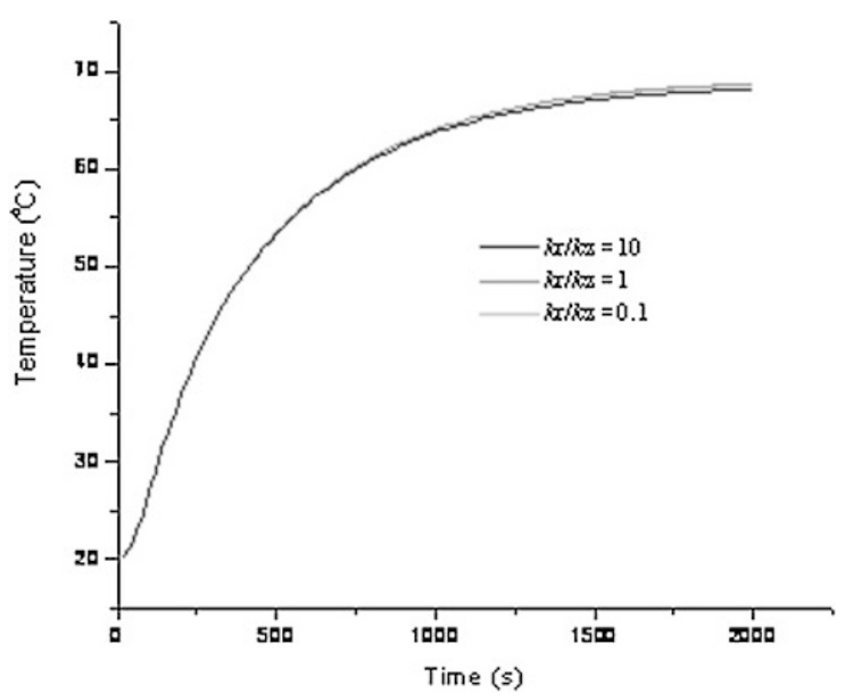

Figure 6. Variation of transient temperature of node 61 with ratio $\lambda_{r} / \lambda_{\mathrm{z}}$. 


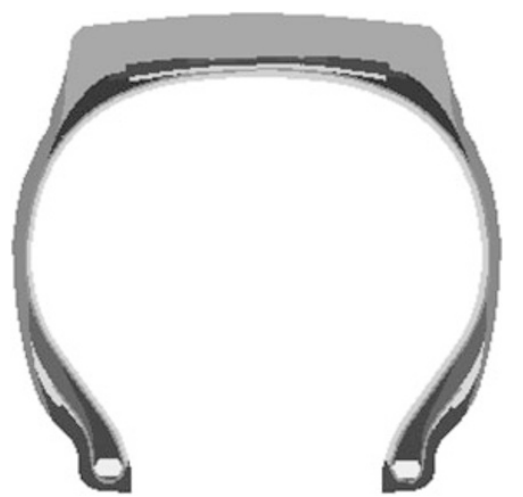

Figure 7. The sectional geometry and material distribution of the truck tire.

node 61 as ratio $\lambda_{r} / \lambda_{\mathrm{z}}$ varies. It can be seen from Figures 5 and 6 that regardless of size of $\lambda_{r} / \lambda_{z}$, agreement among transient temperatures of nodes 6,61 and 116 is very good and the numerical results indeed are not dependent on ratio $\lambda_{r} / \lambda_{\mathrm{z}}$, which proves the accuracy of the finite element formulation presented in this paper.

\section{RESULTS AND DISCUSSIONS}

Over the last decades, the finite element method has been well developed and widely recognized as the most powerful tool for stress and heat transfer analysis. In the present study, we develop an-house simulator for the tire curing process. The simulator employs the finite element method of an axisymmetric heat transfer problem for composite materials as the backbone algorithm. It addresses most of the modeling aspects of tire curing process as mentioned earlier. In this section, some results of the numerical simulation of truck tire curing process will be reported.

The sectional geometry and material distribution of the truck tire studied in the present paper are shown in Figure 6. Its finite element mesh consists of 305 node points and 275 2-D axisymmetric elements, as shown in Figure 7. Figure 8 shows temperature profiles of the media during the tire curing process.

\section{Simulation of Temperature Field}

The nonlinear, transient heat transfer analysis is carried out using the mesh shown in Figure 7. Figures 8 and 9 show a serious of temperature profiles at some time points. It can be seen form Figure 10 that during the heating cycle, heat flows into the tire and that at the end of the heating cycle, almost the entire tire reaches the mold temperature. Figure 11 shows that during the cooling cycle, heat gradually flows out from the tire and that the low diffusivity of rubber compounds keeps a substantial amount of heat inside

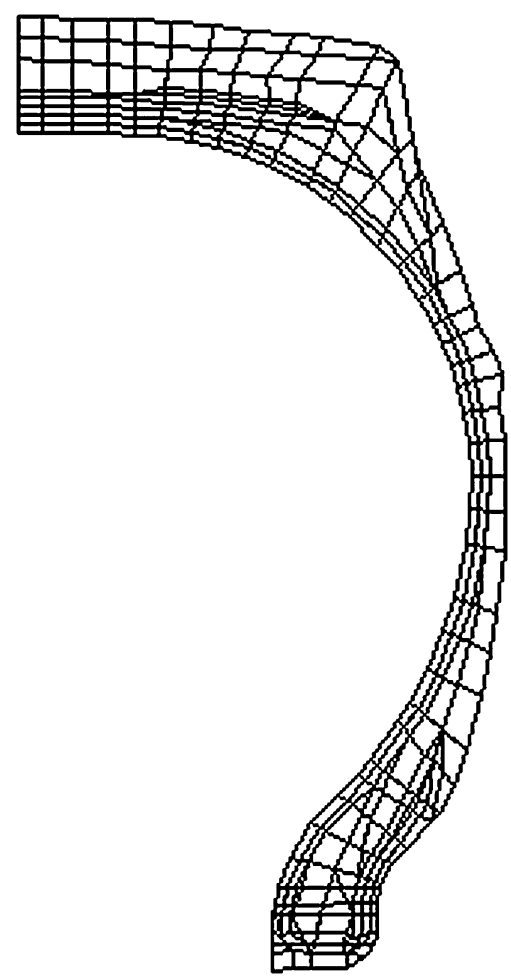

Figure 8. Finite element mesh.

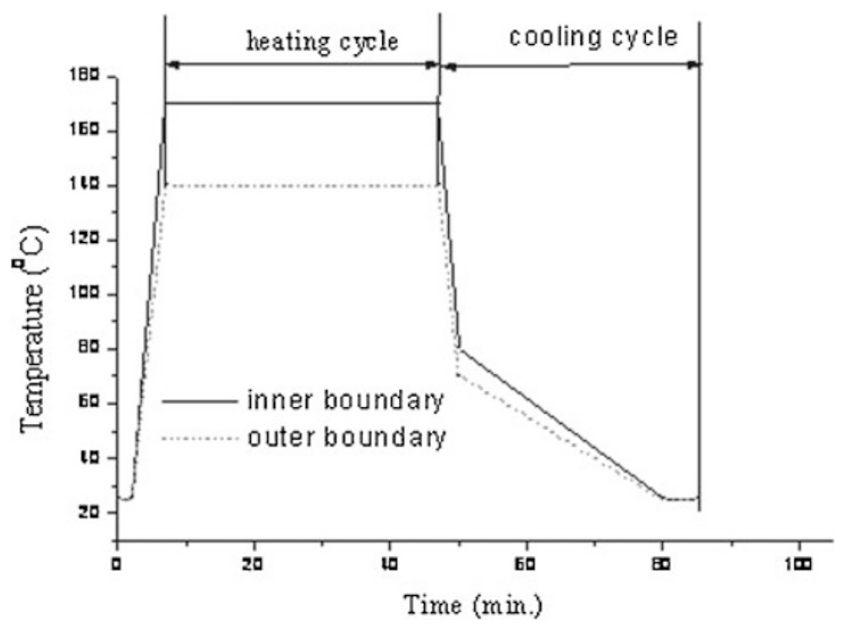

Figure 9. Temperature profiles of the media during the tire curing process.

the tire, especially the thicker part of the tire such as the shoulder and bead areas. Such heat plays an important role in post-curing for truck tires and engineering tires.

\section{Simulation of Cure Extent Field}

Figures 12 and 13 show a serious of SOC profiles at some time points. As far as the tire studied in this paper is concerned with, it can be seen that thicker part such as the shoulder and bead areas reaches proper cure extent much slower than that at thinner part 


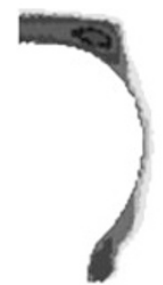

(a) $1000 \mathrm{~s}$

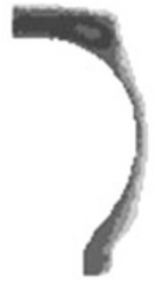

(b) $1500 \mathrm{~s}$

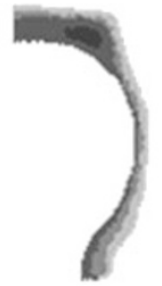

(c) $2000 \mathrm{~s}$

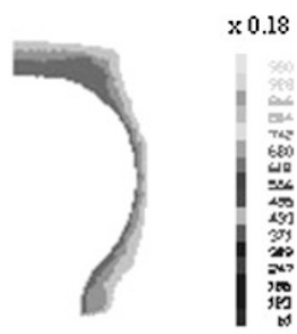

(d) $2700 \mathrm{~s}$

Figure 10. Temperature profiles of the entire tire during the heating cycle.

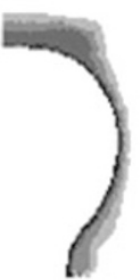

(a) $2820 \mathrm{~s}$

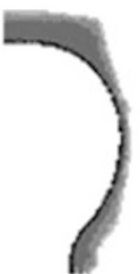

(b) $2940 \mathrm{~s}$

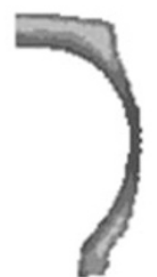

(c) $3190 \mathrm{~s}$

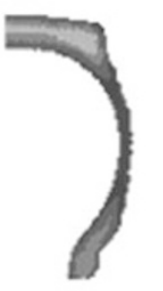

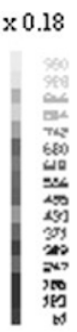

(d) $3640 \mathrm{~s}$

Figure 11. Temperature profiles of the entire tire during the cooling cycle.

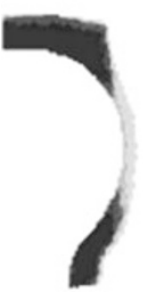

(a) $1000 \mathrm{~s}$

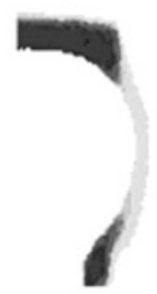

(b) $1500 \mathrm{~s}$

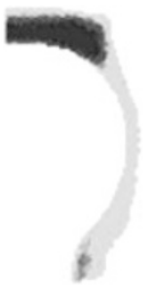

(c) $2000 \mathrm{~s}$

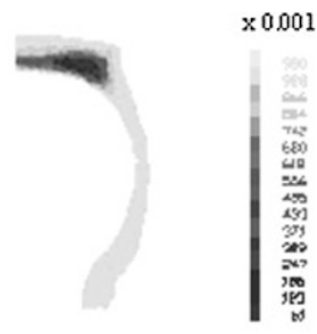

(d) $2700 \mathrm{~s}$

Figure 12. Cure extent profiles of the entire tire during the heating cycle.

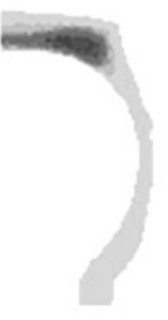

(a) $2820 \mathrm{~s}$

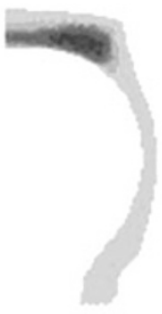

(b) $2940 \mathrm{~s}$

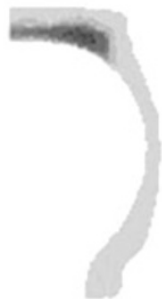

(c) $3190 \mathrm{~s}$

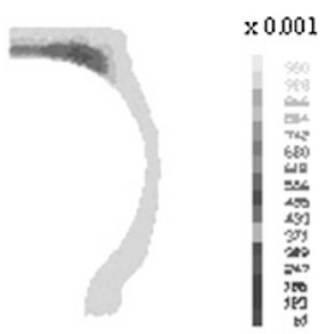

(d) 3640 s

Figure 13. Cure extent profiles of the entire tire during the cooling cycle.

(for example, sidewall). The obvious difference is the reason for over-cure or under-cure at some parts in tire. In addition, it is seen from Figure 13 that a substantial amount of degree of cure is accomplished in the post-curing (or cooling cycle) in the thicker part of the tire.
Simulation of Temperature and State of Cure at Some Typical Points in Tire

In order to indicate the variation of the temperature and the state of cure with time in the tire curing process, the variation of the temperature and the state of cure with time at some typical points in the tire are 




Figure 14. Schematic of the locations of some typical points.

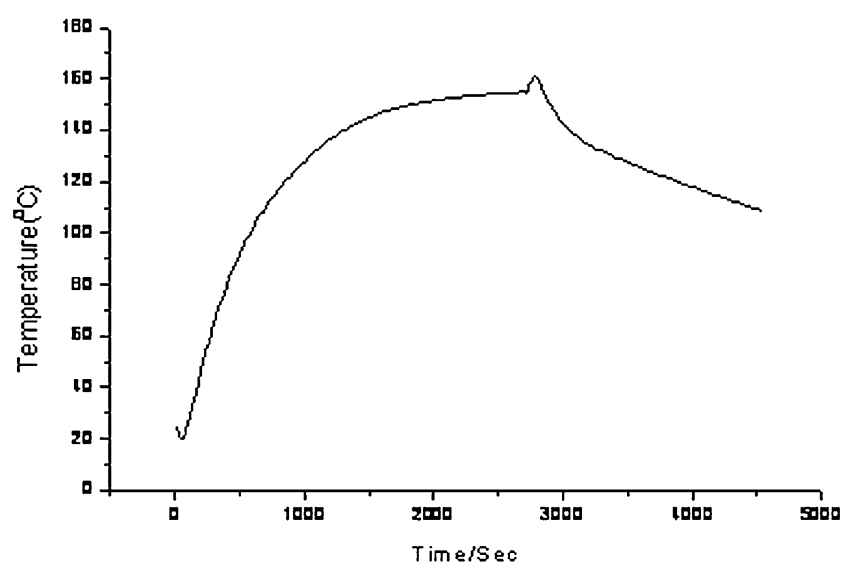

Figure 15. Variation of the temperature with tire curing time at shoulder point $a$.

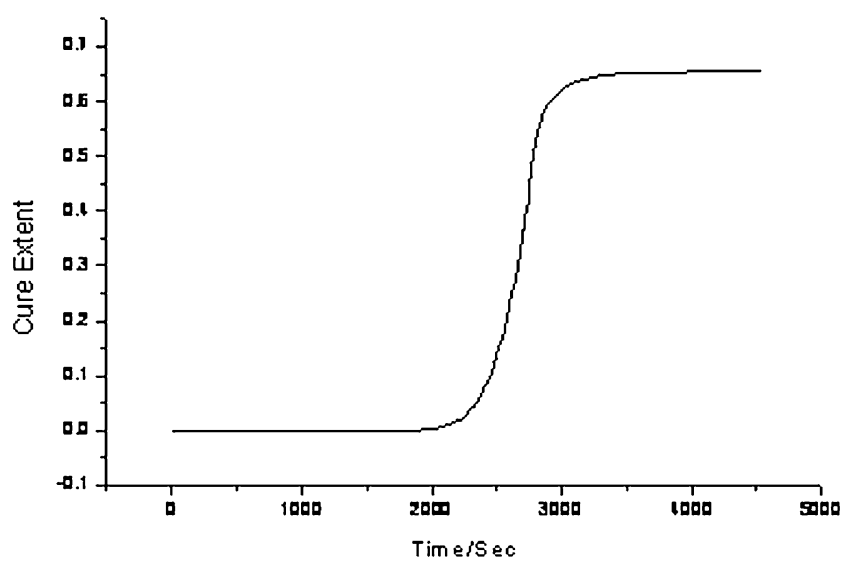

Figure 16. Variation of the state of cure with tire curing time at shoulder point $a$.

also given. Figure 14 shows the locations of some typical points studied in this paper. Points $a, b$ and $c$ in Figure 14 are located around shoulder center, sidewall center and hump center.

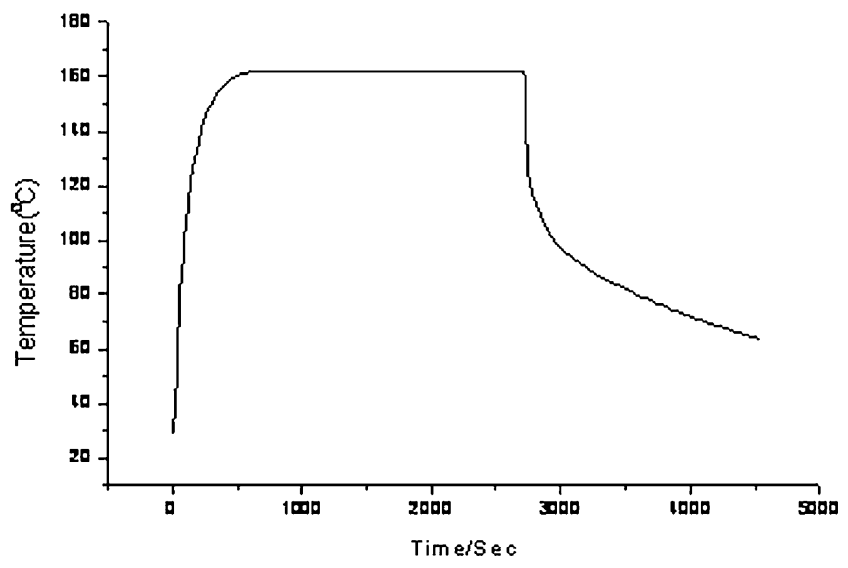

Figure 17. Variation of the temperature with tire curing time at sidewall point $b$.

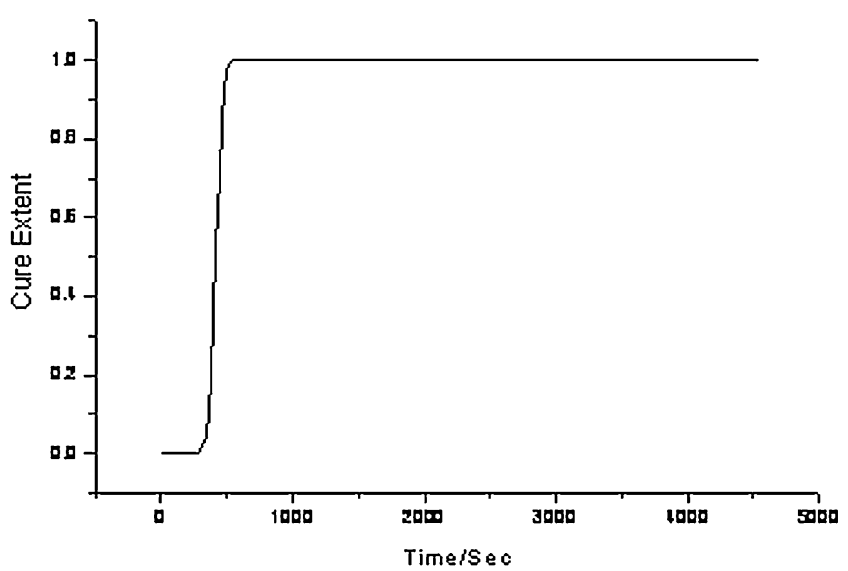

Figure 18. Variation of the state of cure with tire curing time at sidewall point $b$.

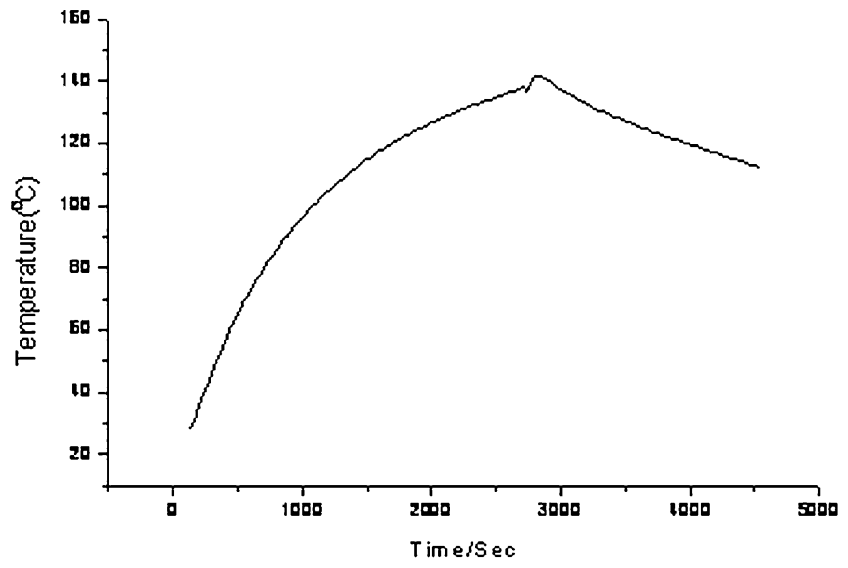

Figure 19. Variation of the temperature with tire curing time at hump point $c$.

Figures 15 and 16 show the variation of the temperature and the state of cure with tire curing time at point $a$ in Figure 14, respectively. Figures 17 and 18 show the variation of the temperature and the state of cure with tire curing time at point $b$ in Figure 14, respectively. Figures 19 and 20 show the variation 


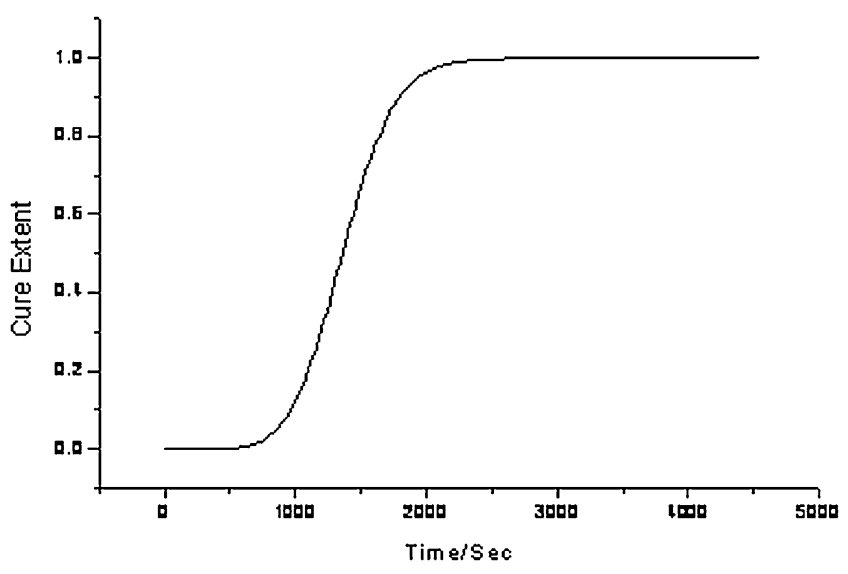

Figure 20. Variation of the state of cure with tire curing time at hump point $c$.

of the temperature and the state of cure with tire curing time at point $c$ in Figure 14, respectively.

It can be seen from Figures 15 and 16 that because the tire thickness at point $a$ is the most thick in the tire, the temperature at point $a$ rises slowly and when boundary heat medium is removed, the temperature profile falls a little, then it rises again sooner. This means that the shoulder point $a$ don't cure completely when heat flux is stopped, and as a result, cure reaction at this point goes on and the heat from cure reaction makes temperature profile rise continuously. The cure profile at point $a$ tells that shoulder point $a$ begins cure reaction quite late and cure reaction doesn't complete. The cure degree of shoulder point $a$ doesn't reach 1. All these are from two points: low diffusivity of rubber compounds and the largest thickness at shoulder.

It can be seen from Figures 17 and 18 that the tire thickness at sidewall point $b$ is quite small compared with the one at shoulder, the temperature profile at this point rises very quickly. As a result, sidewall point $b$ begins cure reaction much earlier than the shoulder point $a$ and reaches proper cure extent quite quickly. In addition, we can see that the temperature profile at this point descends very quickly when boundary heat supply is stopped.

Temperature profile and cure degree profile at hump point $c$ are very similar with those at shoulder point $a$ because the tire at hump point $c$ has also larger thickness.

\section{CONCLUDING REMARKS}

A systematic finite element approach for simulating tire vulcanization has been presented. A simulator for the tire curing processes is developed based. The anisotropy of heat transfer properties of composite mate- rials, the dependence of properties of rubber compounds on the temperature and/or the extent of cure, the time-varying boundary conditions, which include the cooldown of the tire out of the press and the rigorous cure kinetic models, are taken into account. The numerical simulation results of a truck tire curing process show that the simulator successfully describes the trends in temperature and the state of cure during the tire curing process. These are very valuable information for modifying the mold temperatures and compound properties to a more balanced degree of cure. Thus, it also serves as the core module of an optimization algorithm, which is being developed for the tire curing and rubber formulation designs. By the way, it is pointed out that this study is a part of our effort to apply finite element analysis techniques in tire modeling in order to develop the high performance tires. ${ }^{9-20}$

Acknowledgment. Special thanks are due to the National Natural Science Foundation of China (No. 10272037 and No. 10672046) for supporting the present work.

\section{REFERENCES}

1. E. R. G. Eckert and R. M. Drake, in "Analysis of heat and mass transfer," McGraw-Hill, 1972, p 1.

2. O. C. Zienckiewitz, in "The finite element method," New York: McGram-Hill, 1977.

3. J. C. Ambelang and G. A. Prentice, Rubber Chem. Technol., 45, 1195 (1972).

4. G. A. Prentice and M. C. Williams, Rubber Chem. Technol., 53, 1023 (1980).

5. H. P. Schlanger, Rubber Chem. Technol., 56, 304 (1983).

6. G. D. Hubbard and G. M. Simpson, presented at International Rubber Conference, Venice, 1979.

7. W. J. Toth, J. P. Chang, and C. Zanichelli, Tire Sci. Technol., 19, 178 (1991).

8. I. S. Han, C. B. Chung, and J.-H. Kim, Tire Sci. Technol., 24, 50 (1996).

9. X. Yan, Tire Sci. Technol., 29, 186 (2001).

10. X. Yan, Math. Comput. Simulat., 58, 51 (2001).

11. X. Yan, J. Reinf. Plast. Compos., 28, 733 (2003).

12. X. Yan, Y. Wang, and X. Feng, Math. Comput. Simulat., 59, 471 (2002).

13. X. Yan, C. J. Appl. Mech., 19, 106 (2002).

14. X. Yan, Acta Mater. Compos. Sinica, 21, 133 (2001).

15. X. Yan and Y. Wang, et al., Tire Industry, 20, 270 (2000).

16. X. Yan and Y. Wang, et al., Tire Industry, 20, 463 (2000).

17. X. Yan, Acta Mater. Compos. Sinica, 21, 108 (2001).

18. X. Yan, Solid Mechanics Sinica, 22, 150 (2001).

19. X. Yan and D. Wu, et al., Tire Industry, 20, 527 (2000).

20. X. Feng and X. Yan, et al., J. Reinf. Plas. Compos., 23, 373 (2004). 